\title{
A TEST OF HEBB'S POSTULATE AT IDENTIFIED SYNAPSES WHICH MEDIATE CLASSICAL CONDITIONING IN APLYSIA ${ }^{1}$
}

\author{
THOMAS J. CAREW, ${ }^{2}$ ROBERT D. HAWKINS, THOMAS W. ABRAMS, AND ERIC R. KANDEL \\ Center for Neurobiology and Behavior, College of Physicians and Surgeons, Columbia University, and New York State \\ Psychiatric Institute, New York, New York 10032
}

Received July 11, 1983; Revised December 27, 1983; Accepted January 12, 1984

\begin{abstract}
In 1949, D. O. Hebb proposed a novel mechanism for producing changes in the strength of synapses that could account for associative learning. According to Hebb, the strength of a synapse might increase when the use of that synapse contributes to the generation of action potentials in a postsynaptic neuron. Thus, an essential feature of this postulate is that action potentials must occur in both a postsynaptic cell and a presynaptic cell for associative synaptic changes to occur. We have directly tested Hebb's postulate in Aplysia at identified synapses which are known to exhibit a temporally specific increase in efficacy during a cellular analogue of differential conditioning. We find that the mechanism postulated by Hebb is neither necessary nor sufficient to produce the associative change in synaptic strength that underlies conditioning in Aplysia. In contrast, impulse activity in the presynaptic cell must be paired with facilitatory input, supporting the hypothesis that the temporal specificity of classical conditioning in Aplysia can be accounted for by activitydependent amplification of presynaptic facilitation.
\end{abstract}

In $1949, \mathrm{D} . \mathrm{O}$. Hebb proposed a specific mechanism for producing functional changes at synapses that could contribute to associative learning. Hebb suggested that alterations in the strength of synapses might occur when the use of those synapes is associated with and contributes to the generation of action potentials in the postsynaptic neuron. Specifically, Hebb proposed that: "When an axon of cell $\mathrm{A}$ is near enough to excite a cell $\mathrm{B}$ and repeatedly or persistently takes part in firing it, some growth process or metabolic change takes place in one or both cells such that A's efficiency, as one of the cells firing B, is increased."

Although Hebb's original postulate was focused on developmental plasticity such as would occur during the formation of "cell assemblies," it has had a major impact on thinking about the neuronal mechanisms of learning (see for example Marr, 1969; Stent, 1973; Woody et al., 1978; Rauschecker and Singer, 1981; Bienenstock et al.,

\footnotetext{
${ }^{1}$ This work was supported by Career Awards 5K02 MH0081 (to T. J. C.) and 5K05 MH18558 (to E. R. K.), Fellowship 5T32 NS07062-06 (to T. W. A.), and Grants NS 12744 and GM 23540, all from the National Institutes of Health, and by a grant from the McKnight Foundation. We thank Drs. V. Castellucci, G. Clark, I. Kupfermann, and $\mathrm{K}$. Weiss for helpful comments on an earlier draft of this manuscript, L. Katz and K. Hilten for illustrations, and H. Ayers for preparation of the manuscript.

${ }^{2}$ Present address: Department of Psychology, Yale University, P. O. Box 11A, Yale Station, New Haven, CT 06520.
}

1982; Heidmann and Changeaux, 1982). This impact derives from the simple solution Hebb's model offered for two central problems in classical conditioning. First, Hebb's postulate accounts for temporal specificity, the hallmark of associative learning, using plausible physiological mechanisms. Second, it accounts for stimulus and response specificity, which are common features of classical conditioning, without the need of complex neural circuitry.

Hebb envisioned that the most likely physiological mechanism giving rise to the increased synaptic strength was a growth of synaptic "knobs." Thus, although he allowed for other possibilities, Hebb favored the notion that the locus for the plastic changes was presynaptic. In an interesting extension of Hebb's original postulate, Stent (1973) and, more recently, Heidmann and Changeux (1982) have suggested that the synaptic alteration at a Hebb synapse could involve a change in the sensitivity or the state of activation of the receptors of the postsynaptic cell.

An essential feature of Hebb's original postulate is that, for the associative changes to occur, use of a synapse must contribute to the generation of action potentials in a postsynaptic neuron. This aspect of Hebb's postulate has been tested in a variety of neuronal analogues of learning. In some of these studies, postsynaptic activity has been shown to play no role in synaptic plasticity (Kandel and Tauc, 1965; Wurtz et al., 1967). On the other hand, other studies have suggested that postsyn- 
aptic activity may play an important role (O'Brien et al., 1977; Baranyi and Feher, 1981a, b; see also Rauschecker and Singer, 1981). However, in no case has it been possible to relate the change in synaptic efficacy to a specific behavioral instance of associative learning.

Recently, classical conditioning of a simple withdrawal reflex in Aplysia has been demonstrated (Carew et al., $1981,1983)$, and a specific associative change has been localized to an identified set of synapses between the sensory and motor cells for the reflex (Hawkins et al., 1983a). We have now examined whether the associative change at these synapses depends upon the pairing of presynaptic and postsynaptic activity. We found that the mechanism postulated by Hebb does not contribute to classical conditioning of this withdrawal reflex: impulse activity in the postsynaptic cell is neither necessary nor sufficient to produce the temporally specific change in synaptic efficacy that underlies classical conditioning in Aplysia. By contrast, impulse activity in the presynaptic cell is critical. These results are consistent with our earlier finding that the temporal specificity of classical conditioning of this reflex may be explained by activitydependent amplification of presynaptic facilitation (Hawkins et al., 1983a; see also Walters and Byrne, 1983), which is an elaboration of the same mechanism that underlies behavioral sensitization of this reflex (Castellucci and Kandel, 1976; Castellucci et al., 1980; Klein and Kandel, 1978, 1980; Klein et al., 1982).

\section{Materials and Methods}

Aplysia californica weighing 100 to 300 gm were obtained from Pacific Biomarine (Venice, CA). Animals were anesthetized with isotonic $\mathrm{MgCl}_{2}$ (50\% body weight) prior to surgery. The central nervous system (the pedal, pleural, cerebral, and abdominal ganglia and connectives) was dissected free from the body along with the tail nerves (posterior pedal nerves-P9). The ganglia were then pinned to the Sylgard floor of a Lucite recording chamber, and the nerves from the tail were pinned around $\mathrm{Ag}-\mathrm{AgCl}$ stimulating electrodes in the chamber. The abdominal ganglion, which contains the siphon sensory and motor neurons, was partially desheathed, and the preparation was washed with normal artificial seawater (Instant Ocean) for approximately $45 \mathrm{~min}$ before the experiment. We obtained intracellular recordings using standard techniques (Byrne et al., 1974). A Wheatstone bridge circuit was used for recording and passing current simultaneously. Two siphon sensory neurons in the LE cluster (Byrne et al., 1974) were impaled with glass microelectrodes (5 to 15 megohms, filled with 2.5 $\mathrm{M} \mathrm{KCl}$ ) and caused to fire controlled trains of six action potentials by injecting individual $60-\mathrm{msec}$ depolarizing current pulses at a rate of $12 / \mathrm{sec}$ for $0.5 \mathrm{sec}$. In addition, a siphon motor neuron which received monosynaptic excitatory connections from both of the sensory neurons was impaled (see Fig. 1). In a cellular analogue of the differential conditioning procedure (Carew et al., 1983; Hawkins et al., 1983a), action potentials in one sensory neuron were temporally paired with tail nerve stimulation, while action potentials in the other sensory neuron were specifically unpaired. Thus, the action potentials in the two sensory neurons served as the paired and unpaired conditioned stimuli $\left(\mathrm{CS}^{+}\right.$and $\mathrm{CS}^{-}$, respectively), and stimulation of the tail nerve (5-msec biphasic current pulses at $10 \mathrm{~Hz}$ for $1.5 \mathrm{sec}$ ) served as the unconditioned stimulus (US). Current intensity for the tail shock was set to be just supra-threshold for the production of EPSPs in the motor neuron in response to a single (5msec) pulse. In some experiments, action potentials produced directly in the postsynaptic motor neuron by injecting a prolonged $(1.0 \mathrm{sec})$ intracellular depolarizing paired and unpaired sensory neurons were counterbalanced across experiments. The EPSPs from both sensory neurons were measured 15 min after training and compared to their respective pretraining amplitudes (for details see Hawkins et al., 1983a).

Statistical analyses were carried out by means of a $t$ test for either correlated means (within-group comparison) or independent means (between-group comparison). All comparisons were two-tailed. All data are reported as means \pm SEM.

\section{Results}

To explore the role of postsynaptic activity in producing temporally specific changes in synaptic efficacy, we applied to individual nerve cells a differential training procedure based upon the behavioral training procedure that produces differential classical conditioning in Aplysia (see "Materials and Methods"). In previous experiments (Hawkins et al., 1983a) this training procedure produced greater facilitation of the EPSP from the paired sensory neuron than from the unpaired neuron. This effect was attributed to differential amplification of presynaptic facilitation of the paired sensory neuron. However, differential facilitation might also have been due to Hebb-type synaptic plasticity since the unconditioned stimulus (shock to the tail or tail nerve), in addition to causing presynaptic facilitation, also produced excitatory synaptic input in the postsynaptic siphon motor neuron (via a polysynaptic pathway; see Walters et al., 1983), causing it to fire action potentials.

To determine the role of postsynaptic activity in conditioning, we explored whether this activity was either sufficient or necessary for associative changes to occur.

Is postsynaptic activity sufficient for associative changes to occur? To examine this question, we first paired activity in one sensory neuron with a train of action potentials produced directly in the postsynaptic neuron by injecting a prolonged depolarizing current pulse in the motor neuron (see Fig. 2). Strictly speaking, Hebb's postulate requires that the paired $\left(\mathrm{CS}^{+}\right)$neuron "takes part in firing" the postsynaptic (motor) neuron. In our experiments the $\mathrm{CS}^{+}$neuron produced subthreshold depolarization of the motor neuron, but firing of the motor neuron by the US did not occur until after the $\mathrm{CS}^{+}$ stimulation was over. We chose this paradigm because it matches the paradigm that produces conditioning of the withdrawal reflex (Carew et al., 1981, 1983) and associative changes at the sensory neuron-motor neuron synapses (Hawkins et al., 1983a). By contrast, simultaneous presentation of the $\mathrm{CS}^{+}$and the US, which would be more consistent with a strict interpretation of Hebb's 


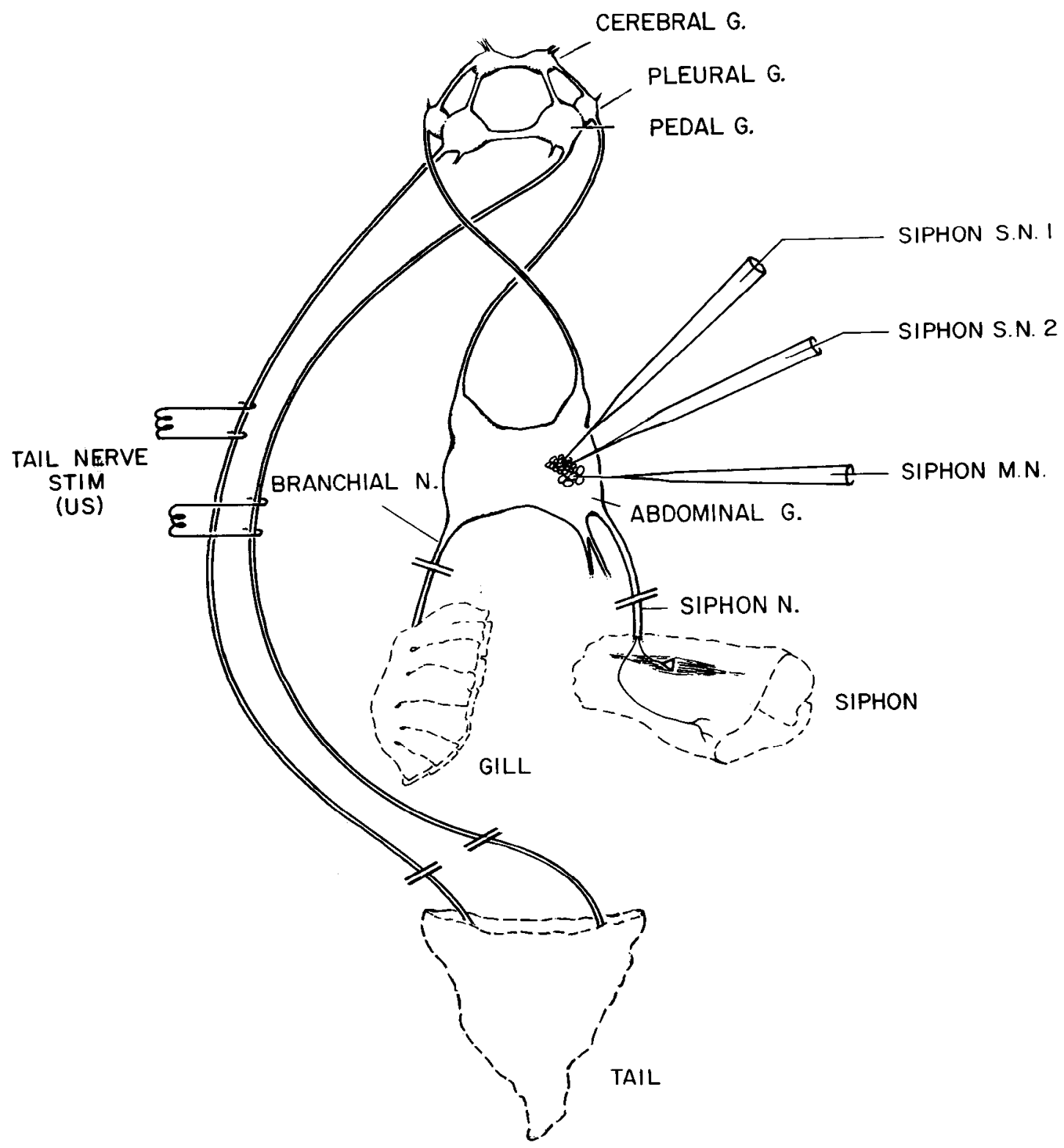

Figure 1. Schematic diagram of experimental preparation. The central nervous system of Aplysia was dissected free and pinned out in a Iucite recording chamber. Two siphon sensory neurons and an identified siphon motor neuron were simultaneously impaled in the abdominal ganglion: one sensory neuron served as $\mathrm{CS}^{+}$, the other as $\mathrm{CS}^{-}$(see the text for details). Nerves from the tail (posterior pedal nerves) were pinned around stimulating electrodes in the chamber to provide the US input. Dashed lines indicate the target organs that the various nerves innervate.

postulate, does not produce good conditioning of this reflex (Hawkins et al., 1983b). Thus the central question of these experiments was whether a Hebb-type mechanism contributes to the associative changes we have observed previously in the behavior and in the synaptic connections. The number and frequency of action potentials produced in the motor neuron (about 10 to 15 spikes in $1.5 \mathrm{sec}$ ) were chosen to approximate the response of that neuron to tail nerve stimulation. On each of five trials, with an intertrial interval of $5 \mathrm{~min}$, the paired sensory neuron was caused to fire a train of action potentials, and this was followed immediately by action potentials in the motor neuron. The other (unpaired) sensory neuron was caused to fire an identical number of action potentials $2.5 \mathrm{~min}$ later. This phase of the experiment was followed by a test (test I) $15 \mathrm{~min}$ after training; then a second phase was run in which activity in each sensory neuron was either paired or unpaired with tail nerve stimulation (Fig. 2). Following this second training phase, a second test (test II) was performed.

An example of the results is shown in Figure 3 . The EPSPs from the paired and unpaired sensory neurons were of roughly equivalent amplitudes prior to training (pretest: $8.7 \mathrm{mV}$ and $8.4 \mathrm{mV}$, respectively). Following five trials with postsynaptic action potentials as the US, the EPSPs from paired and unpaired sensory neurons were both reduced in amplitude by about $45 \%$ (test I: from 8.7 to $5.0 \mathrm{mV}$ and from 8.4 to $4.4 \mathrm{mV}$, respectively). Thus, both sensory neurons showed synaptic depression produced by repeated stimulation; neither showed en- 

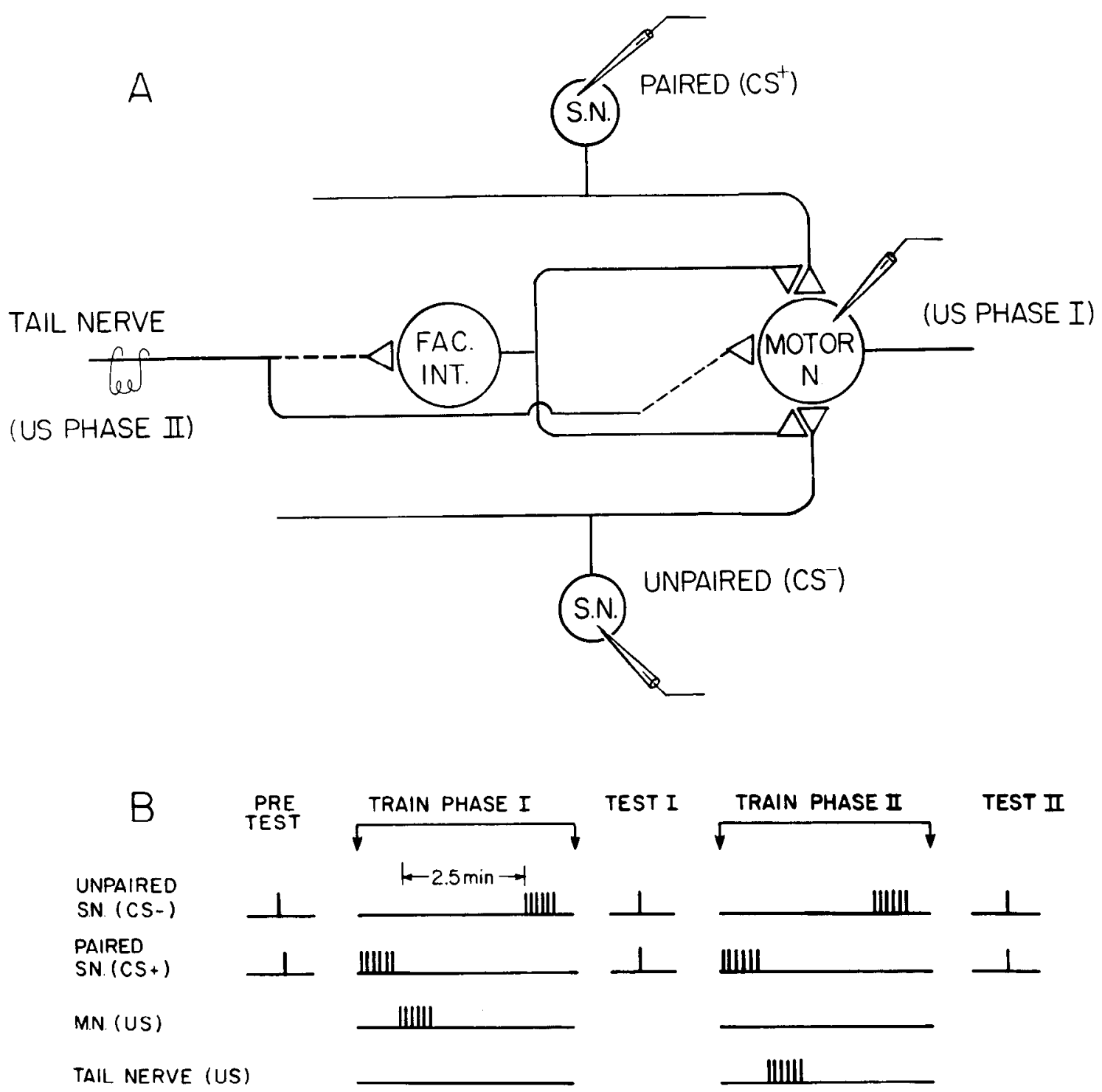

Figure 2. Protocol of experiment examining the sufficiency of postsynaptic activity as a US. $A$, Diagram of experimental arrangement. Two siphon sensory neurons (S.N.) and a siphon motor neuron (M.N.) were simultaneously impaled. Stimulation of each SN produced a monosynaptic EPSP in the M.N. $B$, Diagram of protocol. In phase I of the experiment, activity in one S.N. $\left(\mathrm{CS}^{+}\right)$was paired with action potentials produced directly in the motor neuron with depolarizing current (US-phase I) while activity in the other S.N. $\left(\mathrm{CS}^{-}\right)$was specifically unpaired. In phase II, activity in the S.N.s was either paired or unpaired with tail nerve stimulation (US-phase II).

hanced synaptic transmission. However, following five more training trials in phase II, in which tail nerve stimulation was used as the US, a clear differential facilitation of the EPSP from the paired sensory neuron was observed (test II). Thus, the EPSP from the paired sensory neuron increased from $5.0 \mathrm{mV}$ (test I) to 11.2 $\mathrm{mV}$ (test II), whereas the EPSP from the unpaired sensory neuron increased substantially less, from $4.4 \mathrm{mV}$ (test I) to $5.6 \mathrm{mV}$ (test II). One can directly compare the EPSPs from these paired and unpaired sensory neurons by expressing their test amplitudes as a percentage of their respective pre-training amplitudes. Thus, although EPSPs from both paired and unpaired neurons declined when postsynaptic stimulation served as the US (to $57 \%$ and $52 \%$ of pretraining amplitude, respectively), when tail nerve stimulation was used as the US, the EPSP from the paired sensory neuron was facilitated to $128 \%$ of its pretraining amplitude, whereas the EPSP from the unpaired neuron was only $66 \%$ of its pretraining amplitude.

A summary of 10 experiments is shown in Figure 4. As this graph illustrates, intracellularly produced activity in the postsynaptic neuron produced no facilitation of EPSP amplitude from either the paired or unpaired sensory neurons. Rather, during the five trials in which action potentials in the postsynaptic cell served as reinforcement, both EPSPs showed progressive synaptic depression so that $15 \mathrm{~min}$ after training (test I), their mean amplitude was only about $50 \%$ of their pretraining control amplitude. Thus, postsynaptic activity was not sufficient to produce differential facilitation of the EPSP. By contrast, in eight experiments, when the train- 


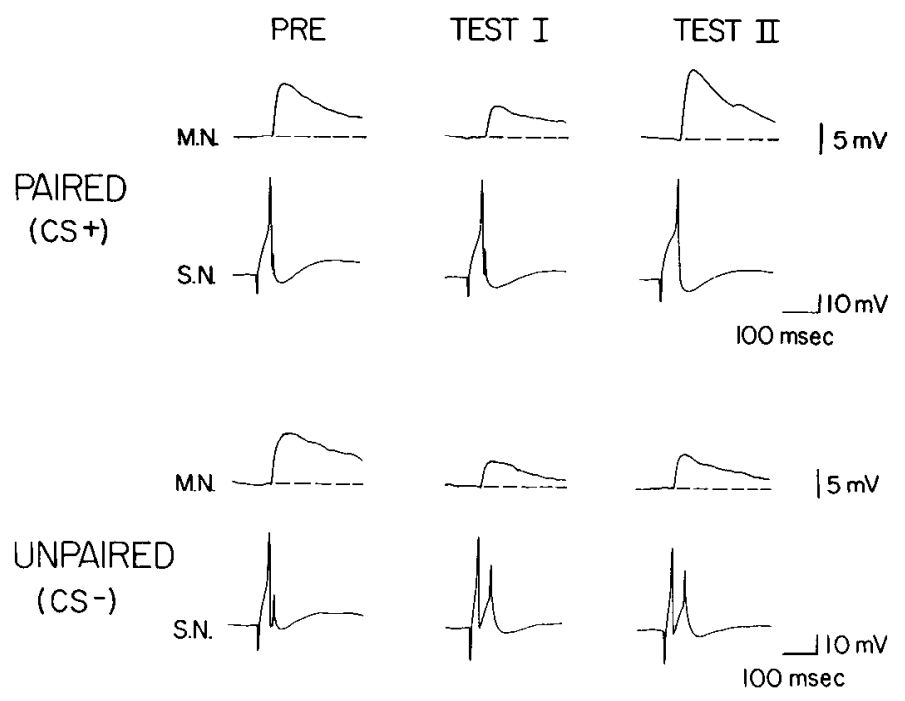

Figure 3. Results of an experiment examining the sufficiency of postsynaptic activity as a US. $P R E$, The amplitudes of the EPSPs from paired ( $C S+$ ) and unpaired (CS-) sensory neurons (S.N.s) onto the motor neuron (M.N.) are approximately equal (8.75 and $8.44 \mathrm{mV}$, respectively). TEST I, Following five trials with motor neuron activity as a US, both EPSPs show no facilitation; rather they both show synaptic decrement (to 5.0 and $4.3 \mathrm{mV}$, respectively). TEST II, When tail nerve stimulation is used as the US, differential facilitation is produced in the paired S.N. compared to the unpaired one $(11.25 \mathrm{mV}$ compared to $5.62 \mathrm{mV}$; see the text and Fig. 3 for details). This experiment was carried out in artificial seawater containing 6 times normal $\mathrm{Ca}^{2+}(66 \mathrm{mM})$ and 3 times normal $\mathrm{Mg}^{2+}(165 \mathrm{mM})$ to reduce contributions of interneurons to the EPSP.

ing procedure was switched so that tail nerve stimulation served as the US, there was clear differential facilitation of the EPSPs from paired sensory neurons compared to those from unpaired sensory neurons (in 2 of the 10 experiments the impalement of one of the three nerve cells was lost during the second phase). In the 15-min test after this second phase of the experiment (test II), the EPSP from the paired neuron was significantly facilitated to a mean of $151 \%$ of its own pretraining amplitude $\left(t_{7}=3.52, p<0.025\right)$; by contrast, the EPSP from the unpaired neuron was only $47 \%$ of its own pretraining amplitude. Thus, at the same synapses where directly produced postsynaptic activity was ineffective as a US, stimulation of the tail nerve produced clear differential facilitation of synaptic transmission.

Is postsynaptic activity necessary for associative changes to occur? Since tail nerve stimulation resulted in brisk trains of action potentials in the siphon motor neuron, it is possible that this postsynaptic activity was necessary for the differential facilitation of the EPSPs from the paired sensory neuron observed in the previous experiment. To test this possibility, the postsynaptic siphon motor neuron was held hyperpolarized during training so that it did not fire action potentials in response to the US. As in phase II of the previous experiment, on each of the five trials (with an intertrial interval of $5 \mathrm{~min}$ ), the sensory neuron that was chosen to be paired with the US was caused to fire a train of action potentials which was immediately followed by a train of electrical pulses to the tail nerves. The other (unpaired) sensory

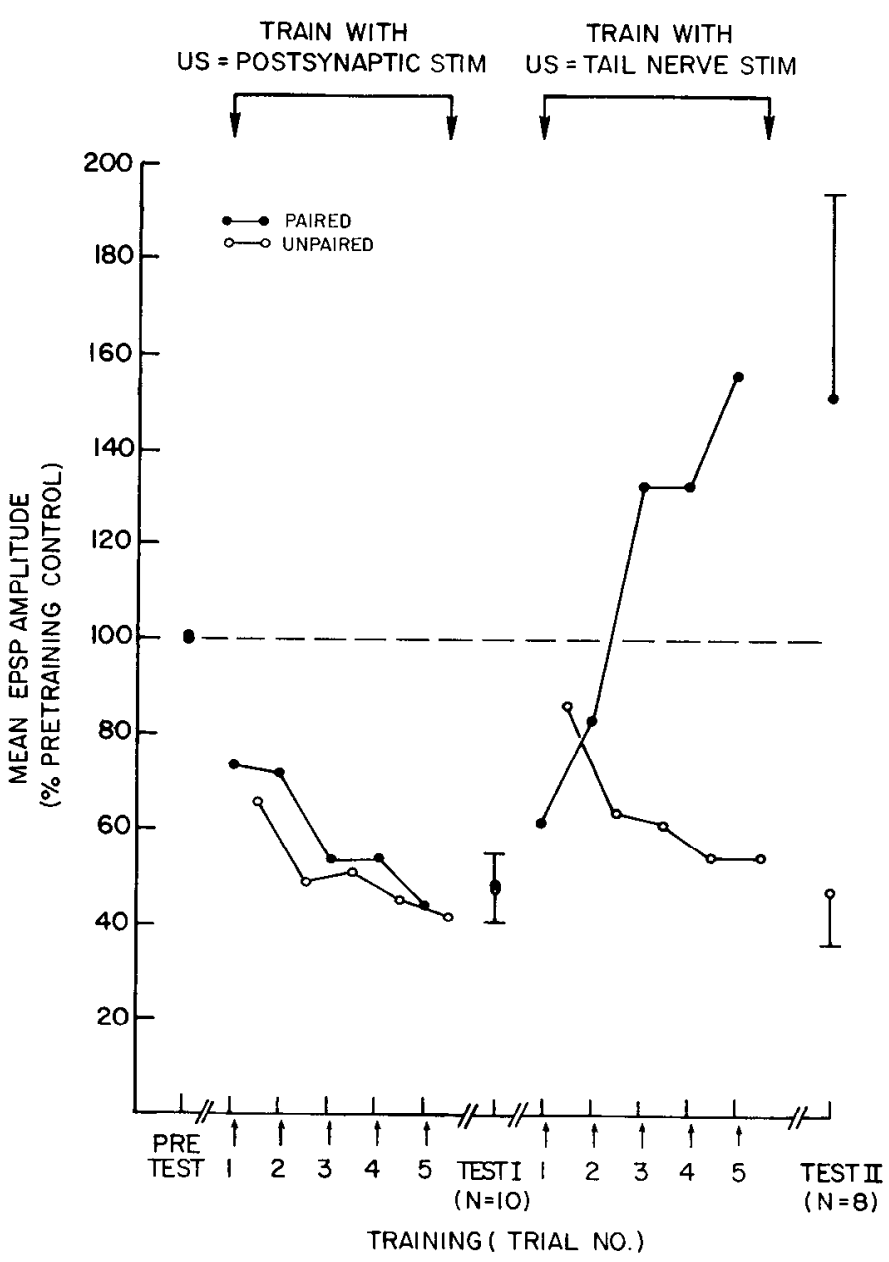

Figure 4. Summary of experiments examining the sufficiency of postsynaptic activity as a US. Training with postsynaptic stimulation as the US (upward arrows, phase I) produces no activity-dependent enhancement; rather, synaptic decrement is produced to about $50 \%$ of control (dashed line) for both paired and unpaired sensory neurons (Test $I$ ). In contrast, when tail nerve stimulation is used as the US (upward arrows, phase II), significant differential facilitation is produced (TEST II: $p<$ 0.025 ). All experiments shown in this figure as well as in Figure 5 were carried out in normal artificial seawater.

neuron was caused to fire an identical number of action potentials 2.5 min later. Early in training the amplitudes of both sensory neuron EPSPs began to increase, although the EPSPs from the paired sensory neurons tended to increase more (Fig. 5). However, later in training, the EPSP from the unpaired sensory cell declined below control level, whereas the EPSP from the paired sensory neuron remained substantially above control. When tested $15 \mathrm{~min}$ after the last training trial, the EPSP from the paired neuron was significantly facilitated compared to its own pretraining amplitude (mean $=174.6 \%$ of pretraining control, $t_{9}=3.55, p<0.025$ ), whereas the EPSP from the unpaired sensory neuron was not facilitated (mean $=85.9 \%$ of pretraining control).

Differential facilitation of synaptic transmission could also be assessed by comparing the difference between facilitation of EPSPs from paired and unpaired sensory neurons within the same experiment. This was done by 


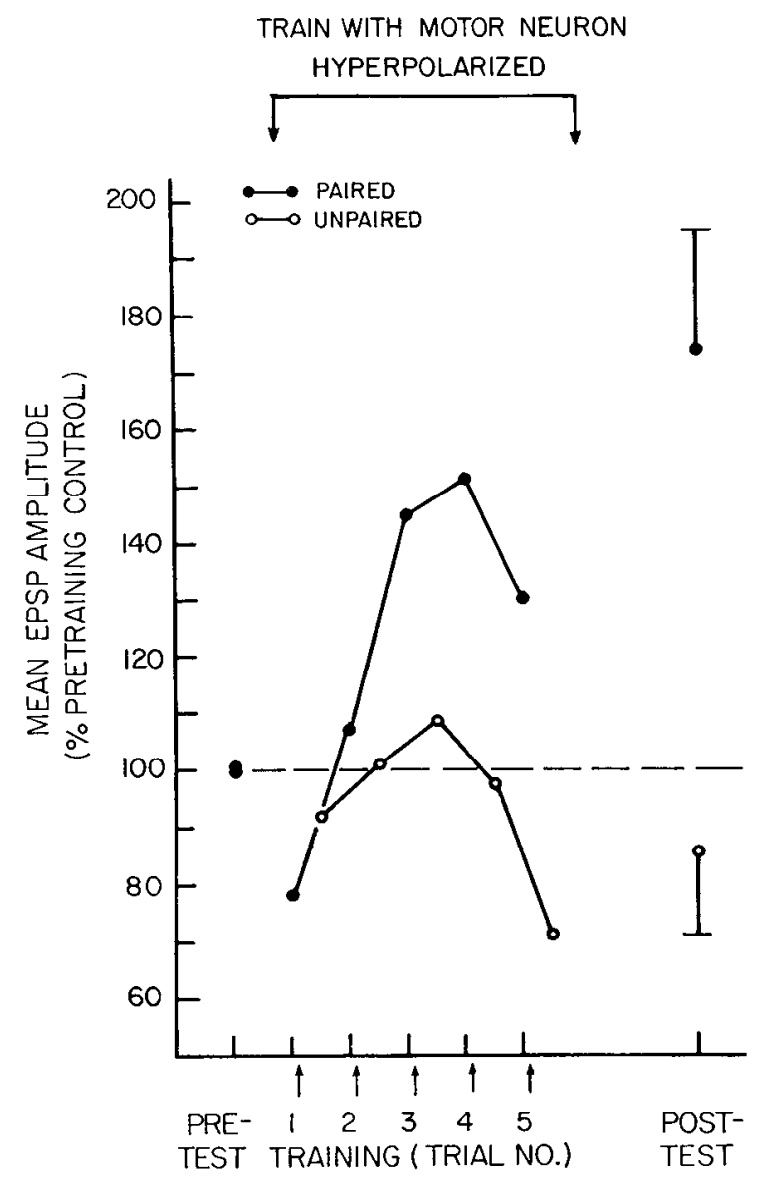

Figure 5. Summary of experiments examining the necessity of postsynaptic activity during conditioning. During training the motor neuron was hyperpolarized so that it fired no action potentials in response to the US (upward arrows). Nonetheless there was significant differential facilitation of the EPSPs from the paired sensory neurons compared to unpaired $(p<0.025)$.

subtracting the normalized value (test scores expressed as a percentage of prescores) for facilitation of the unpaired EPSP from the normalized value for the paired EPSP. Thus, each experiment $(N=10)$ contributed a single score reflecting the difference in facilitation of the EPSPs from paired and unpaired sensory neurons. This comparison revealed significant differential facilitation of the EPSP from the paired sensory neuron (mean difference between paired and unpaired EPSPs $=88.7 \%$; $\left.t_{9}=3.35, p<0.025\right)$. Since the postsynaptic neuron was held hyperpolarized and not permitted to fire during training or testing, these results indicate that postsynaptic activity is not necessary to produce temporally specific associative changes in synaptic efficacy.

\section{Discussion}

Hebb's postulate and classical conditioning in Aplysia. We have attempted to test directly Hebb's postulate for associative learning (Hebb, 1949) by examining an identified set of synapses known to undergo associative changes that contribute to classical conditioning of the siphon and gill-withdrawal reflex in Aplysia. We have found that the pairing of impulse activity in the postsynaptic cell with activity in the presynaptic cell is not critical for the production of the temporally specific change in synaptic effectiveness that accompanies the learning. Confirming previous results, we have found that pairing of activity in the presynaptic cell with facilitatory input is critical for associative changes to occur (Hawkins et al., 1983a).

Hebb's postulate and the model we have proposed based on our results nonetheless share three important features. (1) Both models emphasize that learning involves a change in efficacy of previously existing synaptic connections. (2) Both emphasize that associative learning need not require special neuronal circuitry since the mechanism of temporal specificity can reside in individual neurons. (3) Both emphasize that neuronal activity per se plays an essential role in the cellular mechanisms underlying the formation of temporal associations. The principal difference between the two models centers upon which neurons must be active to give rise to the associative change. Hebb proposed that learning might require coincident activity in both the presynaptic and postsynaptic cell, and he suggested that activity in one cell, the postsynaptic cell (such as might be caused by the US), would be critical for plastic changes in another cell, the presynaptic neuron (see Fig. 6A). Our data indicate that only the cell undergoing the plastic change need be active in association with activity in the US pathway. Thus, in our system, where the plastic change occurs presynaptically, presynaptic activity, coincident with activation of the US pathway, is critical for temporally specific synaptic facilitation (Fig. 6B). An attractive feature of this model is that it provides a mechanism for the temporal specificity of associative learning which is simple, involving only an amplification, in an activity-dependent way, of the same cellular process (presynaptic facilitation) that underlies sensitization. ${ }^{3}$

Activity-dependent modulation of the $K^{+}$channel could produce changes in postsynaptic as well as presynaptic properties. In the model we have proposed, the mechanism for the associative learning involves changes in the release of transmitter from the sensory neurons (Hawkins et al., 1983a). Thus, activity-dependent enhancement of presynaptic facilitation involves a change in the way one neuron transmits information to other cells. However, this should not be taken to imply that associative learning might not also change postsynaptic or receptive properties such as the input resistance or threshold for spike initiation. We have found that the facilitation of synaptic transmission during conditioning involves a

\footnotetext{
${ }^{3}$ We have focused on the synapses between the sensory (CS) neurons and the motor (CR) neurons of the siphon withdrawal reflex, since alterations in transmission at these synapses will influence the reflex response that we measure during conditioning. We have no information as to whether other synapses in Aplysia show Hebb-type plasticity. For example, the question could be raised whether the synapses between the facilitator (US) neurons and the sensory (CS) neurons might have Hebb-type properties, since our training procedure also involves paired spike activity in those neurons (Fig. $6 B$ ). While we have not studied these synapses, we know that the facilitators do not take part in firing the sensory neurons during our training procedure, since spike activity in the facilitators follows spike activity in the sensory neurons. Thus, Hebb-type plasticity at these synapses (if it exists) could not contribute to the associative changes we observe.
} 

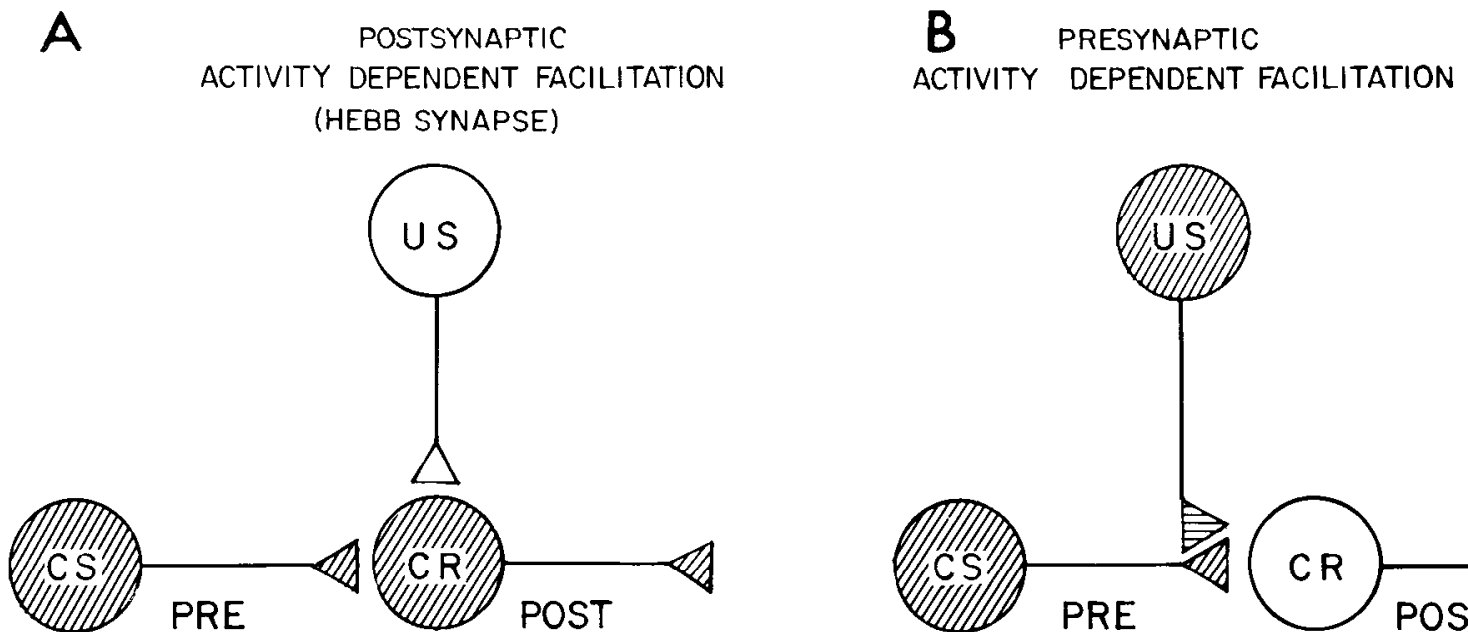
Figure 6 . Comparison of postsynaptic and presynaptic model
in conjunction with presynaptic (CS) activity, for an increase
(CS) activity, paired with facilitator (US) activity, is critical for
for details). Shading indicates that activity is essential.
decrease in $\mathrm{K}^{+}$conductance in the presynaptic sensory
neurons which increases transmitter release by broadening the action potential (Hawkins et al., 1983a). This reduction in $\mathrm{K}^{+}$current also causes an increase in input resistance and a decrease in threshold for spike initiation in the sensory neurons (Klein and Kandel, 1980). Thus, if a similar change in a $\mathrm{K}^{+}$conductance occurred in an interneuron or a motorneuron, in addition to facilitating transmitter release from the terminals of that neuron, it would also increase the efficacy of excitatory synaptic input to that cell (Carew and Kandel, 1976; Schulman and Weight, 1976; Klein and Kandel, 1980; Walters and Byrne, 1983). This modification of these postsynaptic properties would in turn be enhanced by pairing spike activity in the postsynaptic cell with the US input in the same way that presynaptic facilitation is enhanced and thus could contribute to associative learning (for evidence that this type of mechanism may occur in a vertebrate, see Woody et al., 1978). Thus, the critical finding in Aplysia is that only the cell undergoing the plastic change need be active in coincidence with the US pathway for associative learning to occur.

Cases in which receptive (postsynaptic) properties of neurons were modulated would superficially resemble a Hebb-type mechanism, because activity in the postsynaptic cell would be critical to produce the change. However, such cases would not require that presynaptic activity be coincident with postsynaptic activity as Hebb suggested, but would rather require that the postsynaptic action potentials be temporally paircd with facilitatory input. Moreover, this type of plasticity would occur at many synapses onto a cell and not be restricted, as Hebb's postulate would predict, only to the synapses that were activated by the CS input.

In conclusion, although our evidence indicates that Hebb's postulate does not account for classical conditioning of the defensive withdrawal reflex in Aplysia, our results should not be taken to imply that a mechanism such as that proposed by Hebb might not underlie or contribute to other instances of learning. Associative

learning is being studied on the cellular level in several invertebrate and vertebrate preparations (Cohen, 1974; Woolacott and Hoyle, 1977; Davis and Gillette, 1978; Woody et al., 1978; Alkon, 1980; Chang and Gelperin, 1980; Crow and Alkon, 1980; Hoyle, 1980; Sahley et al., 1981; Tsukahara et al., 1981; Alkon et al., 1982; Ito, 1982; McCormick et al., 1982; Walters and Byrne, 1983). It will be of interest to see whether activity-dependent plasticity has a role in learning in these preparations.

\section{References}

Alkon, D. L. (1980) Membrane depolarization accumulates during acquisition of an associative behavioral change. Science 210: 1375-1376.

Alkon, D. L., I. Lederhendler, and J. J. Shoukimas (1982) Primary changes of membrane currents during retention of associative learning. Science 215: 693-695.

Baranyi, A., and O. Feher (1981a) Synaptic facilitation requires paired activation of convergent pathways in the neocortex. Nature 290: 413-415.

Baranyi, A., and O. Feher (1981b) Intracellular studies on cortical synaptic plasticity: Conditioning effect of antidromic activation on test-EPSPs. Exp. Brain Res. 41: 124-134.

Bienenstock, E. L., L. N. Cooper, and P. W. Munro (1982) Theory for the development of neuron selectivity: Orientation specificity and binocular interaction in visual cortex. $J$. Neurosci.. 2: 32-48.

Byrne, J., V. Castellucci, and E. R. Kandel (1974) Receptive field and response properties of Aplysia mechanoreceptor neurons. J. Neurophysiol. 37: 1041-1064.

Carew, T. J., and E. R. Kandel (1976) Two functional consequences of decreased conductance EPSPs: Synaptic augmentation and increased electrotonic coupling. Science 192: 150153.

Carew, T. J., E. T. Walters, and E. R. Kandel (1981) Classical conditioning in a simple withdrawal reflex in Aplysia californica. J. Neurosci. 1: 1426-1437.

Carew, T. J., R. D. Hawkins, and E. R. Kandel (1983) Differential classical conditioning of a defensive withdrawal reflex in Aplysia californica. Science 219: 397-400.

Castellucci, V. F., and E. R. Kandel (1976) Presynaptic facilitation as a mechanism for behavioral sensitization in Aplysia. Science 194: 1176-1178. 
Castellucci, V. F., E. R. Kandel, J. H. Schwartz, F. D. Wilson, A. C. Nairn, and P. Greengard (1980) Intracellular injection of the catalytic subunit of cyclic AMP-dependent protein kinase simulates facilitation of transmitter release underlying behavioral sensitization in Aplysia. Proc. Natl. Acad. Sci. U. S. A. 77: 7492-7496.

Chang, J. J., and A. Gelperin (1980) Rapid taste-aversion learning by an isolated molluscan central nervous system. Proc. Natl. Acad. Sci. U. S. A. 77: 6204-6206.

Cohen, D. H. (1974) The neural pathways and informational flow mediating a conditioning autonomic response. In Limbic and Autonomic Nervous System Research, L. V. DiCara, ed., pp. 223-275, Plenum Press, New York.

Crow, T. J., and D. L. Alkon (1980) Associative behavioral modification in Hermissenda: Cellular correlates. Science 209: $412-414$.

Davis, W. J., and R. Gillette (1978) Neural correlate of behavioral plasticity in command neurons of Pleurobranchaea. Science 199: 801-804.

Hawkins, R. D., T. W. Abrams, T. J. Carew, and E. R. Kandel (1983a) A cellular mechanism of classical conditioning in Aplysia: Activity-dependent amplification of presynaptic facilitation. Science 219: 400-405.

Hawkins, R. D., T. J. Carew, and E. R. Kandel (1983b) Effects of interstimulus interval and contingency on classical conditioning in Aplysia. Soc. Neurosci. Abstr. 9: 168.

Hebb, D. O. (1949) The Organization of Behavior: A Neuropsychological Theory, John Wiley \& Sons, New York.

Heidmann, T., and J. P. Changeaux (1982) Un modele moleculaire de regulation d'efficacite au niveau postsynaptique d'une synapse chimique. C. R. Acad. Sci. Paris 295: 665-670.

Hoyle, G. (1980) Learning, using natural reinforcements, in insect preparations that permit cellular neuronal analysis. J. Neurobiol 11: 323-354.

Ito, M. (1982) Cerebellar control of the vestibular-ocular reflex-Around the flocculus hypothesis. Annu. Rev. Neurosci. 5: 275-296.

Kandel, E. R., and L. Tauc (1965) Mechanism of heterosynaptic facilitation in the giant cell of the abdominal ganglion of Aplysia depilans. J. Physiol. (Lond.) 181: 28-47.

Klein, M., and E. R. Kandel (1978) Presynaptic modulation of voltage dependent $\mathrm{Ca}^{++}$current: Mechanism for behavioral sensitization in Aplysia californica. Proc. Natl. Acad. Sci. U. S. A. 75: 3512-3516.

Klein, M., and E. R. Kandel (1980) Mechanism of calcium current modulation underlying presynaptic facilitation and behavioral sensitization in Aplysia. Proc. Natl. Acad. Sci.
U. S. A. 77: 6912-6916.

Klein, M., J. Camardo, and E. R. Kandel (1982) Serotonin modulates a specific potassium current in the sensory neurons that show presynaptic facilitation in Aplysia. Proc. Natl. Acad. Sci. U. S. A. 79: 5713-5717.

Marr, D. (1969) A theory of cerebellar cortex. J. Physiol. (Lond.) 202: 437-470.

McCormick, D. A., G. A. Clark, D. G. Lavond, and R. F. Thompson (1982) Initial localization of the memory trace for a basic form of learning. Proc. Natl. Acad. Sci. U. S. A. 79 . 2731-2735.

O'Brien, J. H., M. B. Wilder, and C. D. Stevens (1977) Conditioning of cortical neurons in cats with antidromic activation as the unconditioned stimulus. J. Comp. Physiol. Psychol. 91: $918-929$.

Rauschecker, J. P., and W. Singer (1981) The effects of early visual experience on the cat's visual cortex and their possible explanation by Hebb synapses. J. Physiol. (Lond.) 310: 215239.

Sahley C., A. Gelperin, and J. W. Rudy (1981) One-trial associative learning modifies food odor preferences of a terrestrial mollusc. Proc. Natl. Acad. Sci. U. S. A. 78: 640-642.

Schulman, J. A., and F. F. Weight (1976) Synaptic transmission: Long-lasting potentiation by a postsynaptic mechanism. Science 194: 1437-1439.

Stent, G. S. (1973) A physiological mechanism for Hebb's postulate of learning. Proc. Natl. Acad. Sci. U. S. A. 70: 9971001.

Tsukahara, N. Y., Y. Oda, and T. Notsu (1981) Classical conditioning mediated by the red nucleus in the cat. J. Neurosci. 1: 72-79.

Walters, E. T., and J. H. Byrne (1983) Associative conditioning of single sensory neurons suggests a cellular mechanism for learning. Science 219: 405-408.

Walters, E. T., J. H. Byrne, T. J. Carew, and E. R. Kandel (1983) Mechanoafferent neurons innervating the tail of Aplysia. I. Response properties and synaptic connections. J. Neurophysiol. 50: 1522-1542.

Woody, C. D., B. E. Swartz, and E. Gruen (1978) Effects of acetylcholine and cyclic GMP on input resistance of cortical neurons in awake cats. Brain Res. 158: 373-395.

Woolacott, M. H., and G. Hoyle (1977) Neural events underlying learning in insects: Changes in pacemaker. Proc. $\mathrm{R}$. Soc. Lond. Biol. 195: 395-415.

Wurtz, R. H., V. F. Castellucci, and J. Nusrala (1967) Synaptic plasticity: The effect of the action potential in the postsynaptic neuron. Exp. Neurol. 18: 350-368. 